Check for updates

Cite this: RSC Adv., 2019, 9, 22289

\title{
Heat treatment to improve the wear resistance of PTFE/PMMA composites
}

\author{
Dapeng Gu, (D) ab Longxiao Zhang, ${ }^{a}$ Suwen Chen, (DD *c Kefeng Song, ${ }^{a}$ Deng Pan, ${ }^{\text {ab }}$ \\ Bingchao Yang ${ }^{d}$ and Shouyao Liu ${ }^{a}$
}

Polytetrafluoroethylene/poly(methyl methacrylate) (PTFE/PMMA) composites were prepared by a selfcuring method. The influence of heat treatment processes on the friction and wear behaviors of PTFE/ PMMA composites against bearing steel balls were studied by a ball-on-disk tribometer. The thermal performance of PTFE/PMMA composites with heat treatment was analyzed by Thermogravimetric Analysis (TGA). The surface morphologies and element distribution of PTFE/PMMA composites in a PMMA matrix were detected by Field Emission Scanning Electron Microscopy (FE-SEM) and Energy Dispersive Spectroscopy (EDS). The results indicated that the wear rates of PTFE/PMMA composites with different heat treatments significantly declined compared with the unheated treatment composite. The wear rate of the PTFE/PMMA composite decreased firstly and then increased with molding temperature and time increasing, and reduced with the molding pressure increasing. The main wear mechanisms of PTFE/PMMA composites with different heat treatments were fatigue wear and abrasive wear.

Received 11th June 2019 Accepted 13th July 2019

DOI: $10.1039 / c 9 r a 04362 d$

rsc.li/rsc-advances obviously reduce the friction coefficient or wear rate. In addition, the friction and wear of PMMA based composite can be obviously reduced by either filling PTFE alone ${ }^{16}$ or PTFE/Cu/ $\mathrm{Al}_{2} \mathrm{O}_{3}$ in synergetic action. ${ }^{17}$

Heat treatment is also considered as one of the effective methods to improve the mechanical and tribological properties of polymers. ${ }^{18}$ Ayman et al. ${ }^{19}$ found that heat treatment process could improve the tribological properties of polyamide (PI). Wang et al. ${ }^{20}$ studied the effect of different molding pressures on the mechanical and tribological properties of ultra-high molecular weight polyethylene (UHMWPE) and found that the molding pressures were conducive to decrease the friction coefficient and wear rate of UHMWPE. Sarkar et al. ${ }^{21}$ found that the friction coefficient and wear rate of polyamide 6 (PA6) were both decreased by heat treatment. Therefore, the friction coefficient and wear rate of polymer can be reduced by the appropriate heat treatment process.

However, there are few literature studies focusing on the influence of heat treatment process on the tribological performances of PMMA based composites. In this paper, self-curing PTFE/PMMA composites were prepared and the dry sliding tribological behavior of PTFE/PMMA composites with various molding pressures, molding temperatures and molding times were studied.

\section{Experimental}

\subsection{Material}

A commercial acrylic product of PMMA powder was supplied by Arkema, Serquigny, France. The methyl methacrylate (MMA) 
$(\geq 99.5 \%)$ monomer liquid, PTFE $(5 \mu \mathrm{m})$ powder and catechol $(\geq 99.5 \%)$ were all supplied by Aladdin Reagent Co. Ltd., Shanghai, China. The dibenzoyl peroxide (BPO, $\geq 98 \%$; containing water $28-32 \%$ ), $N, N$-dimethyl- $p$-toluidine (Dmpt, 98\%) were both supplied by Sinopharm Group Chemical Reagent Co. Ltd., Shanghai, China.

\subsection{Specimen preparation}

The PMMA matrix was synthesized by self-curing at room temperature. The synthesis process of PMMA based composite was introduced in detail in ref. 16 and 17. In this paper, the mass ratio of the powder mixture to the liquid mixture was $3: 2$ and the mass ratio of PTFE was $25 \mathrm{wt} \%$.

\subsection{Heat treatment}

The PTFE/PMMA composites were secondary molding by means of hot press molding technique. Heat treatment parameters of PTFE/PMMA composites are listed in Table 1. At the end of each run of heat treatment process, the resulting specimens were cooled along with the furnace. The mold for heat treatment of PTFE/PMMA composite was made of steel. The inner diameter of the steel mold was the same as PTFE/PMMA composite fabricated by self-curing.

\subsection{Material characterization}

The worn surfaces of PTFE/PMMA composites were examined by Field Emission Scanning Electron Microscopy (FE-SEM, Sigma 500, Carl Zeiss, Oberkochen, Germany). To establish the degree of PTFE dispersion within the PMMA matrix, the worn and fractured surfaces were observed by FE-SEM and Energy Dispersive Spectroscopy (EDS). Thermal properties of PTFE/PMMA composites were studied by Thermogravimetric Analysis (TGA, STA449C, NETZSCH, Germany). And PTFE/ PMMA composite were heated from $35{ }^{\circ} \mathrm{C}$ to $500{ }^{\circ} \mathrm{C}$ with the temperature increasement rate of $10^{\circ} \mathrm{C} \mathrm{min}^{-1}$ in the presence

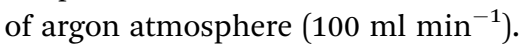

\subsection{Tribology tests}

The friction and wear tests were carried out in a ball-on-disk tribometer (CSM Instruments, Peseux, Switzerland) in which the stationary bearing steel ball was in contact with a rotating polymer disk. The size of the ball (bearing steel, AISI E52100, hardness of HRC $60-65, R_{\mathrm{a}}<0.2 \mu \mathrm{m}$ ) was $6 \mathrm{~mm}$ diameter. The size of PTFE/PMMA composite was $50 \mathrm{~mm}$ diameter and $10 \mathrm{~mm}$ height. Before each test, the ball was cleaned for $30 \mathrm{~min}$ in alcohol using an ultrasonic cleaner. The PTFE/PMMA composites were polished $\left(R_{\mathrm{a}}=0.4-0.6 \mu \mathrm{m}\right)$ by a polishing machine and washed with alcohol and deionized water, then ultrasonic cleaned for $30 \mathrm{~min}$ in deionized water. The PTFE/PMMA composites were performed at ambient temperature with sliding speed of $0.2 \mathrm{~m} \mathrm{~s}^{-1}$, normal load of $5 \mathrm{~N}$ and total sliding distance of $500 \mathrm{~m}$. In order to ensure the correctness of the data, each group of experiment was repeated three times.

At the end of the experiment, the friction coefficient curve with sliding distance could be obtained directly by the CSM tribometer. The average value of the friction coefficient was calculated from five points of relative stationary part. The wear volume was measured by a conscan confocal optical profilometer (Anton Paar Compact Platform Company, Graz, Austria). The wear performance was expressed by the specific wear rate calculated by the following equation,

$$
\omega=\Delta V /(F L)
$$

where $\omega$ is the specific wear rate in $\mathrm{mm}^{3}(\mathrm{~N} \mathrm{~m})^{-1}, \Delta V$ is the volume loss in $\mathrm{mm},{ }^{3} \mathrm{~F}$ is the applied normal load in $\mathrm{N}, L$ is the total sliding distance in $\mathrm{m}$.

\section{Results and discussion}

\subsection{Element distribution analysis}

Fig. 1 shows the SEM photo and the distribution of $\mathrm{C}, \mathrm{F}$ and $\mathrm{O}$ elements by EDS in the section of PTFE/PMMA composites. C element is mainly from PMMA matrix and PTFE. O element mainly comes from PMMA matrix. F element originates from PTFE. According to the distribution of F element, it could be found that the distribution of PTFE was relatively uniform in the PMMA matrix.

\subsection{Thermal properties}

3.2.1. Effect of molding temperatures. The Thermogravimetric (TG) curves of PTFE/PMMA composites under different molding temperatures are shown in Fig. 2. And the results are summarized in Table 2. 5 and $10 \mathrm{wt} \%$ decomposition of PTFE/ PMMA composites were measured and designated as $T_{5}$ and $T_{10}$, respectively. $T_{\mathrm{c}}$ was defined as the complete decomposition temperature. From Fig. 2 and Table $2, T_{5}$ and $T_{10}$ of PTFE/PMMA

Table 1 Heat treatment parameters of PTFE/PMMA composites

\begin{tabular}{|c|c|c|c|}
\hline Test number & Molding pressure/Mpa & Molding temperature $/{ }^{\circ} \mathrm{C}$ & Molding time/min \\
\hline 1 & 10 & 80 & 15 \\
\hline 2 & 10 & 110 & 15 \\
\hline 3 & 10 & 140 & 15 \\
\hline 4 & 10 & 110 & 5 \\
\hline 5 & 10 & 110 & 25 \\
\hline 6 & 5 & 110 & 15 \\
\hline 7 & 15 & 110 & 15 \\
\hline
\end{tabular}



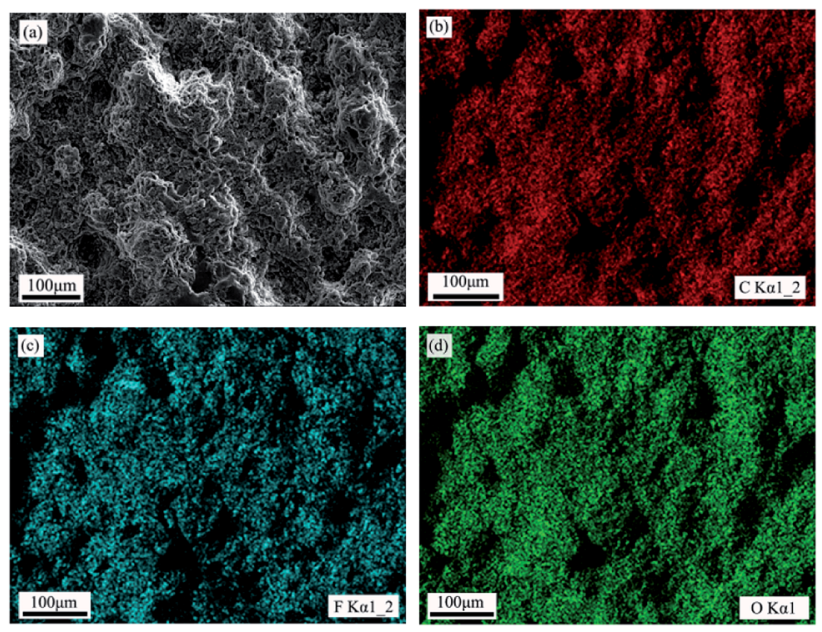

Fig. 1 The section's SEM photo and EDS mappings of characteristic elements for PTFE/PMMA composites under $10 \mathrm{MPa}, 110{ }^{\circ} \mathrm{C}$ and 15 min. (a) SEM; (b) C element; (c) F element; (d) $O$ element.

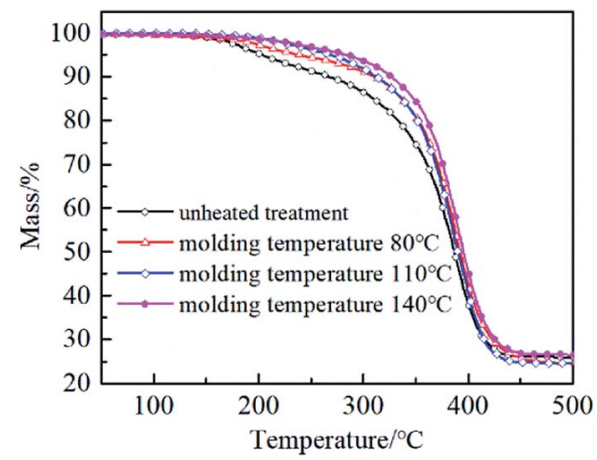

Fig. 2 TG curves of PTFE/PMMA composites under different molding temperatures (molding pressure is $10 \mathrm{MPa}$ and molding time is $15 \mathrm{~min}$ ).

Table 2 Thermal decomposition temperatures of PTFE/PMMA composites under different molding temperatures

\begin{tabular}{llll}
\hline $\begin{array}{l}\text { Molding } \\
\text { temperatures }\end{array}$ & $T_{5}\left({ }^{\circ} \mathrm{C}\right)$ & $T_{10}\left({ }^{\circ} \mathrm{C}\right)$ & $T_{\mathrm{c}}\left({ }^{\circ} \mathrm{C}\right)$ \\
\hline Unheated treatment & 207 & 266 & 444 \\
$80^{\circ} \mathrm{C}$ & 243 & 309 & 448 \\
$110^{\circ} \mathrm{C}$ & 274 & 314 & 450 \\
$140{ }^{\circ} \mathrm{C}$ & 280 & 327 & 453
\end{tabular}

composites under different molding temperatures were in the range of $243-280{ }^{\circ} \mathrm{C}$ and $309-327^{\circ} \mathrm{C}$ respectively, which were significantly higher than that of unheated treatment PTFE/ PMMA composites. This indicates that molding temperatures could improve the thermal stability of PTFE/PMMA composites. Fouad and Pegoretti et al. ${ }^{22,23}$ found that the mechanical properties of crystalline polymers (such as UHMWPE and polyethylene (PE)) were improved by heat treatment process. This enhancement is achieved by increasing crystallinity. For amorphous and self-curing PMMA, the main reasons for the improvement of thermal properties may be the fact that the residual small molecules in PMMA matrix will continue to participate in the polymerization reaction under the action of temperature, which leads to the increase of the molecular weight of PMMA. $T_{5}$ values of PTFE/PMMA composites under different molding temperatures increased with the molding temperature increasing. $T_{5}$ value of PTFE/PMMA composite with molding temperature $140^{\circ} \mathrm{C}$ was $37^{\circ} \mathrm{C}$ higher than that of composite with molding temperature $80{ }^{\circ} \mathrm{C}$. With the increasement of molding temperature, PTFE/PMMA composites gradually transit from glassy state to highly elastic state. And in this process, PTFE particles are more dispersed with the expansion of PMMA matrix, which effectively prevents the diffusion of degradation products in PMMA matrix and slows down the thermal degradation process. ${ }^{24-26}$

3.2.2. Effect of molding times. The TG curves of PTFE/ PMMA composites under different molding times are shown in Fig. 3. And the results are summarized in Table 3. From Fig. 3 and Table 3, molding time had also importance to thermal properties of PTFE/PMMA composites when the molding pressure and temperature were constant. Compared with the unheated treatment PTFE/PMMA composites, $T_{5}$ values of composites under different molding times were $67-80{ }^{\circ} \mathrm{C}$ higher. Within the range of the molding time (5-25 min), the thermal decomposition temperature fluctuated with the molding time increasing. $T_{5}$ and $T_{10}$ values of PTFE/PMMA composites were optimal when the molding time was $5 \mathrm{~min}$.

3.2.3. Effect of molding pressures. The TG curves of PTFE/ PMMA composites under different molding pressures are shown in Fig. 4. And the results are summarized in Table 4. From Fig. 4 and Table 4, the thermal properties of PTFE/PMMA composites could be improved significantly by changing the molding pressure when the molding temperature and time were constant. The largest values of $T_{5}$ and $T_{10}$ of PTFE/PMMA composites were $296{ }^{\circ} \mathrm{C}$ and $337{ }^{\circ} \mathrm{C}$ respectively, under the molding pressure $15 \mathrm{MPa}$.

Compared with the unheated treatment PTFE/PMMA composites, $T_{5}$ and $T_{10}$ values of PTFE/PMMA composites increased about $89{ }^{\circ} \mathrm{C}$ and $71{ }^{\circ} \mathrm{C}$ respectively. This can be explained by the fact that the compactness of the composite becomes better with the molding pressure increasing, and the

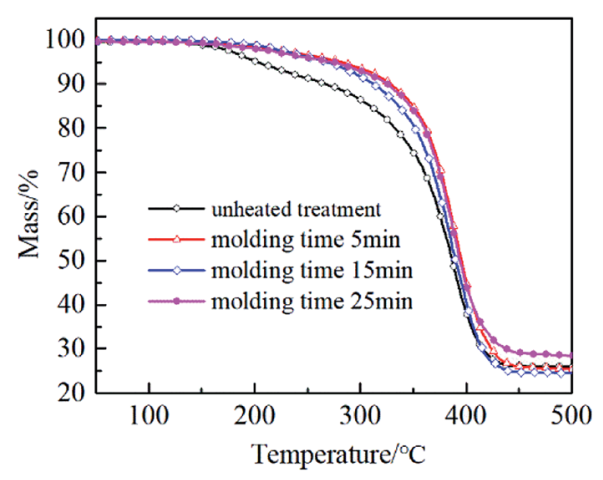

Fig. 3 TG curves of PTFE/PMMA composites under different molding times (molding pressure is $10 \mathrm{MPa}$ and molding temperature is $110^{\circ} \mathrm{C}$ ). 
Table 3 Thermal decomposition temperatures of PTFE/PMMA composites under different molding times

\begin{tabular}{llll}
\hline Molding times & $T_{5}\left({ }^{\circ} \mathrm{C}\right)$ & $T_{10}\left({ }^{\circ} \mathrm{C}\right)$ & $T_{\mathrm{c}}\left({ }^{\circ} \mathrm{C}\right)$ \\
\hline Unheated treatment & 207 & 266 & 444 \\
$5 \mathrm{~min}$ & 287 & 329 & 444 \\
$15 \mathrm{~min}$ & 274 & 314 & 450 \\
$25 \mathrm{~min}$ & 278 & 324 & 453
\end{tabular}

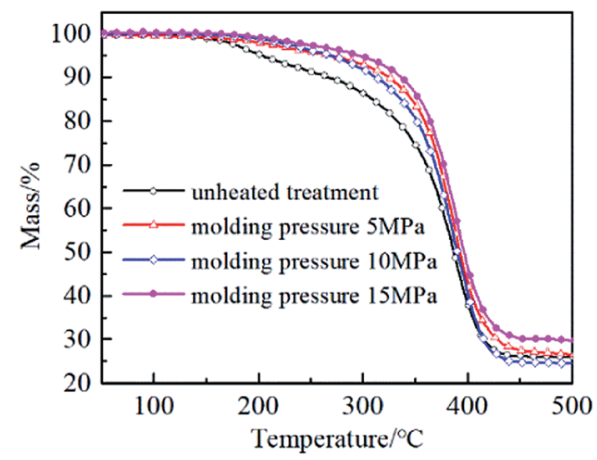

Fig. 4 TG curves of PTFE/PMMA composites under different molding pressures (molding time is $15 \mathrm{~min}$ and molding temperature is $110^{\circ} \mathrm{C}$ ).

Table 4 Thermal decomposition temperatures of PTFE/PMMA composites under different molding pressures

\begin{tabular}{llll}
\hline Molding pressures & $T_{5}\left({ }^{\circ} \mathrm{C}\right)$ & $T_{10}\left({ }^{\circ} \mathrm{C}\right)$ & $T_{\mathrm{c}}\left({ }^{\circ} \mathrm{C}\right)$ \\
\hline Unheated treatment & 207 & 266 & 444 \\
$5 \mathrm{MPa}$ & 270 & 324 & 453 \\
$10 \mathrm{MPa}$ & 274 & 314 & 450 \\
$15 \mathrm{MPa}$ & 296 & 337 & 460
\end{tabular}

interaction force between the long molecular chains in the matrix becomes stronger. ${ }^{20,27}$ Thus the thermal decomposition temperature rose.

\subsection{Friction and wear behaviors}

3.3.1. Effect of molding temperatures. The effects of molding temperatures on the friction coefficient and wear rate of PTFE/PMMA composite are shown in Fig. 5. It is evident from Fig. 5 that the friction coefficients of PTFE/PMMA composites had little difference under various molding temperatures. The friction coefficients of different molding temperatures lain in the range of $0.142-0.145$ and the lowest friction coefficient was observed when the molding temperature was $110^{\circ} \mathrm{C}$. But there were obvious difference about the wear rates of PTFE/PMMA composites with different molding temperatures. The lowest wear rate of PTFE/PMMA composite was about $3.95 \times 10^{-6}$ $\mathrm{mm}^{3}(\mathrm{~N} \mathrm{~m})^{-1}$ under the molding temperature $110^{\circ} \mathrm{C}$. This was obviously lower than the unheated treatment composite. Table 2 shows that the molding temperature improves the thermal properties of PTFE/PMMA composites, which may be the reason for the better wear resistance of heat treated composites. In

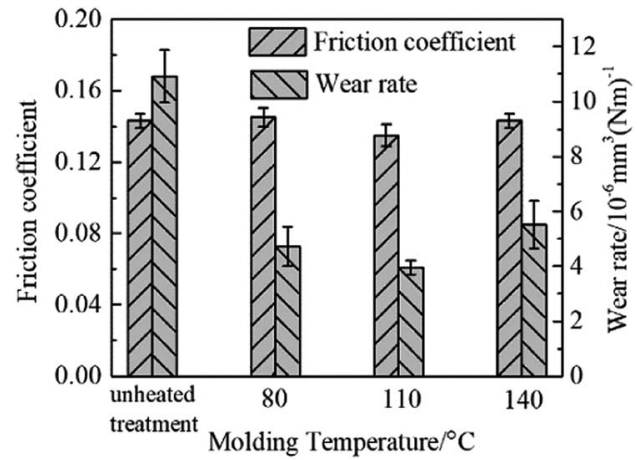

Fig. 5 Effect of the molding temperature on the friction coefficient and wear rate of PTFE/PMMA composite (molding pressure is $10 \mathrm{MPa}$ and molding time is $15 \mathrm{~min}$ ).

addition, the wear rate firstly decreased and then increased within the molding temperature $80-140{ }^{\circ} \mathrm{C}$. Compared with the PTFE/PMMA composites under the molding temperature $80{ }^{\circ} \mathrm{C}$ and $140^{\circ} \mathrm{C}$, the wear rate under the molding temperature $110^{\circ} \mathrm{C}$ decreased about $16 \%$ and $28 \%$, respectively.

3.3.2. Effect of molding times. The effects of molding times on the friction coefficient and wear rate of PTFE/PMMA composite are shown in Fig. 6. From Fig. 6, it is found that the friction coefficients under different molding times of PTFE/ PMMA composites had slight fluctuation and the lowest friction coefficient was about 0.142 under the molding time $15 \mathrm{~min}$. As it can be seen, the smallest wear rate of PTFE/PMMA composite was almost $3.95 \times 10^{-6} \mathrm{~mm}^{3}(\mathrm{~N} \mathrm{~m})^{-1}$ with the molding time 15 min. Compared with PTFE/PMMA composite under the molding time $25 \mathrm{~min}$, the wear rate of PTFE/PMMA composite under the molding time 15 min declined about $35 \%$. Similarly, compared with the unheated treatment PTFE/PMMA composite, the wear rates with different molding times significantly decreased.

3.3.3. Effect of molding pressures. The effect of molding pressure on the friction coefficient and wear rate of PTFE/ PMMA composite is shown in Fig. 7. From Fig. 7, the friction coefficient of PTFE/PMMA composite decreased slightly with the molding pressure increasing. The friction coefficient with

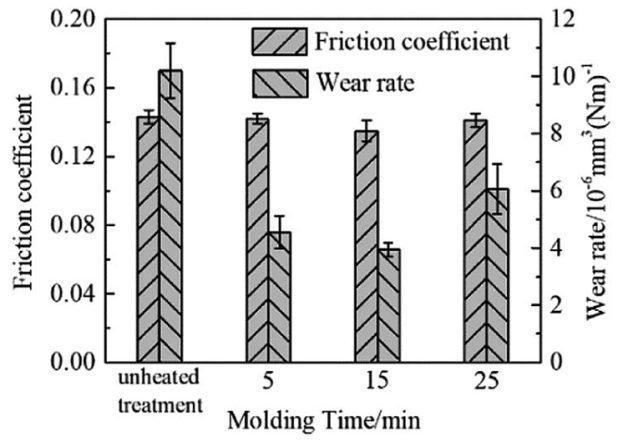

Fig. 6 Effect of the molding time on the friction coefficient and wear rate of PTFE/PMMA composite (molding temperature is $110{ }^{\circ} \mathrm{C}$ and molding pressure is $10 \mathrm{MPa}$ ). 


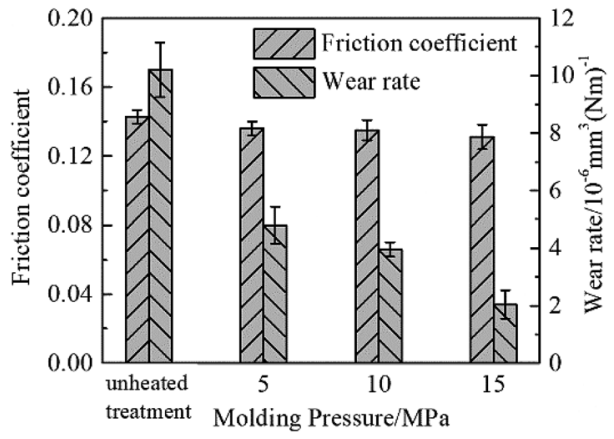

Fig. 7 Effect of the molding pressure on the friction coefficient and wear rate of PTFE/PMMA composite (molding temperature is $110^{\circ} \mathrm{C}$ and molding time is $15 \mathrm{~min})$.

the molding pressure $15 \mathrm{MPa}$ was relatively low, showing an average value 0.140 . It is seen that the wear rate of PTFE/PMMA composite could obvious reduce with the molding pressure increasing. The lowest wear rate was $2.04 \times 10^{-6} \mathrm{~mm}^{3}(\mathrm{~N} \mathrm{~m})^{-1}$ under the molding pressure $15 \mathrm{MPa}$ and decreased approximately 58\% comparing with that under the molding pressure $5 \mathrm{MPa}$. The main reason is that the hardness ${ }^{20}$ and thermal stability of the composite increase with the molding pressure, so the PTFE/PMMA composite performed better wear resistance.

\subsection{SEM analysis}

The typical morphologies of the worn surfaces of PTFE/PMMA composites under different heat treatments were investigated by SEM, as shown in Fig. 8. It can be seen that there are many circular areas with different sizes on the PTFE/PMMA composite surfaces. In order to determine the compositions of those circular areas, EDS of typical elements were recoded and the results were shown in Fig. 8(f). It indicates that those circular areas are PMMA matrix. Meanwhile, it can be seen that the circular areas tended to become larger with the molding temperature and time increasing. Ref. 16 has proved that pure PMMA has poor wear resistance. When more PMMA matrix was exposed, the wear rates of composites began to increase. This may be the reason that the wear rate increased when the molding temperature is high $\left(140{ }^{\circ} \mathrm{C}\right)$ and the molding time is long (25 min). Fig. 8(a), (c) and (f) show that the worn surfaces of PTFE/PMMA composite are accompanied by severe abrasive wear, which correspond to the relatively high wear rate. While Fig. 8(b) and (f) show a relatively stable worn surface with only slight scratch, which indicates that there is slight wear during the wear process of PTFE/PMMA composite. This is consistent with the results of the lower friction coefficient and wear rate described above (Fig. 5-7). However, Fig. 8(b) (molding pressure $10 \mathrm{MPa}$ ) shows many spalling on the worn surfaces of PTFE/ PMMA composites. It indicates that adhesion wear occurs on the worn surface. And this eventually leads to the wear rate higher than Fig. 8(g) (molding pressure $15 \mathrm{MPa}$ ). From Fig. 8(a)$(\mathrm{g})$, there are some cracks on the worn surfaces. This indicates
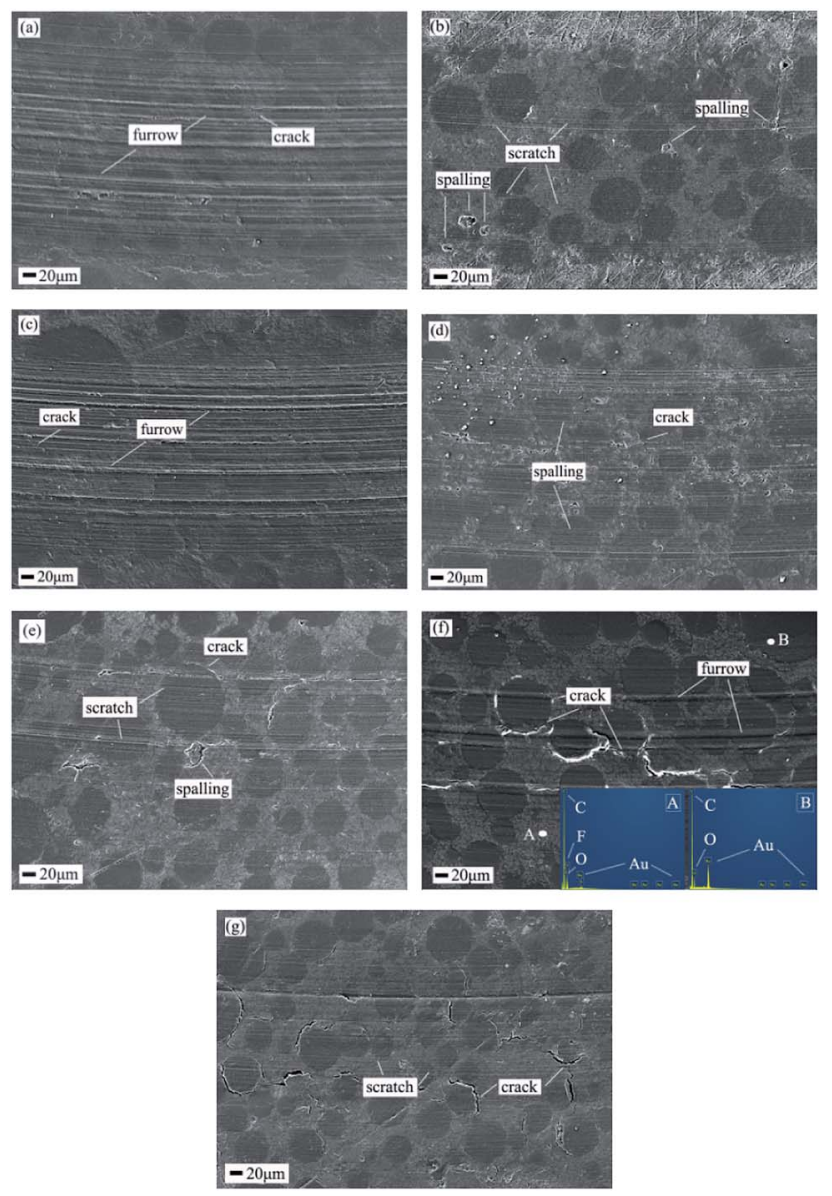

Fig. 8 SEM pictures of the representative worn surface morphologies for PTFE/PMMA composites under different molding pressures, temperatures and times. (a) $10 \mathrm{MPa}, 80^{\circ} \mathrm{C}$, and $15 \mathrm{~min}$; (b) $10 \mathrm{MPa}$, $110{ }^{\circ} \mathrm{C}$ and $15 \mathrm{~min}$; (c) $10 \mathrm{MPa}, 140{ }^{\circ} \mathrm{C}$ and $15 \mathrm{~min}$; (d) $10 \mathrm{MPa}, 110{ }^{\circ} \mathrm{C}$ and $5 \mathrm{~min}$; (e) $10 \mathrm{MPa}, 110^{\circ} \mathrm{C}$ and $25 \mathrm{~min}$; (f) $5 \mathrm{MPa}, 110^{\circ} \mathrm{C}$ and $15 \mathrm{~min}$; (g) $15 \mathrm{MPa}, 110{ }^{\circ} \mathrm{C}$ and $15 \mathrm{~min}$.

that the fatigue wear occurs during the wear process of PTFE/ PMMA composites.

\section{Conclusions}

(1) Compared with the unheated treatment PTFE/PMMA composite, the thermal properties of the composites significantly increased under different heat treatment processes. Within the parameters of heat treatment, the temperature of mass loss 5 wt $\%\left(T_{5}\right)$ increased with the increasing of the molding temperature and pressure, but fluctuated with the molding time increasing.

(2) Compared with the unheated treatment PTFE/PMMA composite, the friction coefficient changed slightly under different heat treatment conditions, but the wear rate significantly decreased.

(3) The wear rate of PTFE/PMMA composite decreased firstly and then increased with the molding temperature and time increasing, and reduced with the molding pressure increasing. When the molding pressure was $15 \mathrm{MPa}$, molding temperature 
was $110{ }^{\circ} \mathrm{C}$ and molding time was $15 \mathrm{~min}$, the wear rate of PTFE/ PMMA composite was the lowest.

(4) The main wear mechanisms of PTFE/PMMA composite under different heat treatments were fatigue wear and abrasive wear. And there was slight adhesive wear.

\section{Conflicts of interest}

There are no conflicts to declare.

\section{Acknowledgements}

This work was supported by National Natural Science Foundation of China (Grant No. 51605418), Natural Science Foundation of Hebei Province (Grant No. E2016203103), Youth Projects of Department of Education of Hebei Province (Grant No. QN2017148).

\section{Notes and references}

1 Z. B. Cai, M. H. Zhu, S. Yang, X. B. Xiao, X. Z. Lin and H. Y. Yu, Wear, 2011, 271, 2242-2251.

2 M. M. Demir, M. Memesa, P. Castignolles and G. Wegner, Macromol. Rapid Commun., 2006, 27, 763-770.

3 S. Gross, D. Camozzo, V. Di Noto, L. Armelao and E. Tondello, Eur. Polym. J., 2007, 43, 673-696.

4 C. Dong, C. Yuan, X. Bai, X. Yan and Z. Peng, RSC Adv., 2014, 4, 19034-19042.

5 C. Gao, G. Zhang, T. Wang and Q. Wang, RSC Adv., 2016, 6, 51247-51256.

6 J. G. Xu, H. B. Yan and D. G. Gu, Mater. Des., 2014, 61, 270274.

7 M. Kalin, M. Zalaznik and S. Novak, Wear, 2015, 332-333, 855-862.

8 V. Pettarin, M. J. Churruca, D. Felhös and K. J. Karger, Wear, 2010, 269, 31-45.
9 L. Chang, Z. Zhang, H. Zhang and A. K. Schlarb, Compos. Sci. Technol., 2006, 66, 3188-3198.

10 L. D. Aguilera-Camacho, C. Hernández-Navarro, K. J. Moreno and J. S. García-Miranda, J. Coat. Technol. Res., 2015, 12, 347355.

11 A. Akinci, S. Sen and U. Sen, Composites, Part B, 2014, 56, 4247.

12 L. Y. Lin and D. E. Kim, Tribol. Int., 2011, 44, 1926-1931.

13 M. Avella, M. E. Errico and E. Martuscelli, Nano Lett., 2001, 1, 213-217.

14 Z. Yang, B. Dong, Y. Huang, L. Liu, F. Y. Yan and H. L. Li, Mater. Lett., 2005, 59, 2128-2132.

15 G. T. Gu, Z. J. Zhang and H. X. Dang, Appl. Surf. Sci., 2004, 221, 129-135.

16 D. P. Gu, L. X. Zhang, S. W. Chen, K. F. Song and S. Y. Liu, Polymers, 2018, 10, 966.

17 D. P. Gu, L. X. Zhang, S. W. Chen, K. F. Song and S. Y. Liu, J. Appl. Polym. Sci., 2018, 135, 46705.

18 A. A. Ayman, Int. J. Mater. Chem. Phys., 2015, 1, 132-140.

19 A. A. Ayman, E. B. Zeidan, A. M. Hamed and W. Y. Ali, EGTRIB Journal of the Egyptian Society of Tribology, 2010, 7, 52-64.

20 S. B. Wang and S. R. Ge, Wear, 2007, 263, 949-956.

21 A. D. Sarkar, Friction and Wear, Academic Press, London, 1980.

22 H. Fouad, A. H. I. Mourad and D. C. Barton, Polym. Test., 2005, 24, 549-556.

23 A. Pegoretti, M. Ashkar, C. Migliaresi and G. Marom, Compos. Sci. Technol., 2000, 60, 1181-1189.

24 T. Kashiwagi, F. Du, J. F. Douglas, K. I. Winey, R. H. Harris and J. R. Shields, Nat. Mater., 2005, 4, 928.

25 L. Chen, Z. Huang, G. Dong, C. He, L. Liu, Y. Hu and Y. Li, Polym. Compos., 2009, 30, 239-247.

26 L. Q. Cui, N. H. Tarte and S. L. Woo, Macromolecules, 2008, 41, 4268-4274.

27 N. C. Prasnis and K. Ramani, J. Mater. Sci., 1998, 9, 165-172. 